\title{
Impact of Actinobacillus pleuropneumoniae biofilm mode of growth on the lipid A structures and stimulation of immune cells
}

Skander Hathroubi ${ }^{1}$, Francis Beaudry ${ }^{2}$, Chantale Provost $^{1}$, Léa Martelet $^{1}$, Mariela Segura ${ }^{1}$, Carl A Gagnon $^{1}$ and Mario Jacques ${ }^{1 *}$

${ }^{1}$ Centre de recherche en infectiologie porcine et avicole, Faculté de médecine vétérinaire, Université de Montréal, St-Hyacinthe, Québec, Canada.

${ }^{2}$ Groupe de recherche en pharmacologie animale du Québec, Faculté de médecine vétérinaire, Université de Montréal, Québec, Canada.

* Corresponding author: Mario Jacques, Ph.D.

Faculté de médecine vétérinaire, Université de Montréal, 3200 rue Sicotte, St-Hyacinthe, Québec, Canada J2S 2M2

Tel: +1 450 773-8521 ext. 8348; Fax: +1 450 778-8108

Email:mario.jacques@umontreal.ca 


\section{ABSTRACT}

Actinobacillus pleuropneumoniae (APP), the etiologic agent of porcine pleuropneumonia, forms biofilms on both biotic and abiotic surfaces. APP biofilms confers resistance to antibiotics. To our knowledge, no studies have examined the role of APP biofilm in immune evasion and infection persistence. This study was undertaken to: (i) investigate biofilm-associated lipopolysaccharide modifications occurring during the switch to biofilm mode of growth; (ii) characterize pro-inflammatory cytokines expression in porcine pulmonary alveolar macrophages (PAMs) and proliferation in porcine peripheral blood mononuclear cells (PBMCs) challenged with planktonic or biofilm APP cells. Extracted lipid A samples from biofilm and planktonic cultures were analyzed by HPLC-high resolution accurate mass spectrometry. Biofilm cells displayed significant changes in lipid A profiles when compared to their planktonic counterparts. Furthermore, in vitro experiments were conducted to examine the inflammatory response of PAMs exposed to UV-inactivated APP grown in biofilm or in suspension. Relative mRNA expression of pro-inflammatory genes IL-1, IL-6, IL-8 and MCP-1 decreased in PAMs when exposed to biofilm cells compared to planktonic cells. Additionally, the biofilm state reduced PBMCs proliferation. Taken together, APP biofilm cells show a weaker ability to stimulate innate immune cells which could be due, in part, to lipid A structure modifications.

KEYWORDS: Actinobacillus pleuropneumoniae, Biofilm, Cytokines, Innate immune cells, Lipid A, Macrophages 


\section{INTRODUCTION}

Biofilms are adherent communities of bacteria attached to biological or non-biological surfaces and encased within a self-produced polymeric matrix that protects cells from noxious elements in the surrounding environment. ${ }^{1,2}$ Biofilm represents a serious therapeutic challenge, since bacteria within biofilm are usually more resistant to antibiotics and to immune clearance..$^{3-6}$ Biofilms are ubiquitous and nearly all microorganisms are able to adhere to surfaces and generate biofilms. This includes Actinobacillus pleuropneumoniae, a Gram-negative porcine pathogen and member of the Pasteurellaceae family. ${ }^{7}$ A. pleuropneumoniae strains can form biofilms on both abiotic ${ }^{7-9}$ and biotic ${ }^{10}$ surfaces. A. pleuropneumoniae cells within biofilms are 100 to 30,000 times more resistant to antibiotics than their planktonic counterparts ${ }^{11}$ and might play an important role in the persistence of bacterial infection in swine.

A. pleuropneumoniae is the etiologic agent of porcine pleuropneumonia, a fibrinous, hemorrhagic, highly contagious disease affecting pigs of all ages and leading to high economic losses in the swine industry. ${ }^{12}$ Symptoms and signs of porcine pleuropneumonia are depression, loss of appetite, fever, and severe breathing difficulties with some coughing. ${ }^{13}$ After death, it is common to see blood discharge out of the nasal cavity due to extensive lung tissue damage. ${ }^{13}$ The disease in its acute form is associated with high rates of morbidity and mortality. ${ }^{13,14}$ Lung tissues are damaged by the RTX toxins, ApxI, Apx II, Apx III and Apx IV. ${ }^{15,16}$ In addition, surface lipopolysaccharides (LPS) induce an inflammatory response that contributes to necrosis of porcine lung epithelial cell. ${ }^{17-19}$ Inflammatory cytokines in lung tissue may exacerbate the effect of A. pleuropneumoniae, and add to host-mediated tissue damage. ${ }^{17,19,20}$ Conversely, subclinical or chronic forms of the disease are asymptomatic, undetectable and less invasive. ${ }^{21,22}$ Chronic infection plays a role in persistence and transmission of several bacteria. ${ }^{19,20}$ Numerous studies 
have also reported that biofilms play a role in immune evasion and bacterial survival. ${ }^{23-25}$ In $A$. pleuropneumoniae, most studies were performed using planktonic cells despite the important role played by biofilms in the pathogenesis of human and animal chronic infection. ${ }^{26-29}$

Nothing is known about how A. pleuropneumoniae biofilm interacts with immune cells, and if lipid A undergoes structural modifications in A. pleuropneumoniae biofilms. The purpose of this study was to (i) investigate modifications of lipid A occurring during the switch to the biofilm mode of growth and (ii) characterize the pattern of inflammatory cytokines expression in porcine pulmonary alveolar macrophages (PAMs) and the proliferative response of porcine peripheral blood mononuclear cells (PBMCs) challenged with A. pleuropneumoniae planktonic and biofilm cells. 


\section{MATERIALS AND METHODS}

\section{Cell line and bacterial strain}

A. pleuropneumoniae MBHPP147 (APP147) is a non-hemolytic derivative of the serotype 1 reference strain S4074. ${ }^{10}$ APP147 contains deletions in both the apxIC and apxIIC genes. Bacterial strain was cultured in brain heart infusion (BHI) broth or agar (Oxoid Ltd, Basingstoke, Hampshire, England) supplemented with $15 \mu \mathrm{g} / \mathrm{mL}$ nicotinamide adenine dinucleotide (NAD) incubated at $37^{\circ} \mathrm{C}$ in $5 \% \mathrm{CO}_{2}$. Porcine pulmonary alveolar macrophage (PAM) 3D4/21 cell line was purchased from the American Type Culture Collection (ATCC CRL-2843) and was maintained in RPMI 1640 medium (Invitrogen, Eugene, OR, USA) with 10\% fetal bovine serum (FBS; heat inactivated) and adjusted to contain $2 \mathrm{mM}$ L-glutamine (Invitrogen), $10 \mathrm{mM}$ HEPES (Invitrogen), $1 \mathrm{mM}$ sodium pyruvate, $1 \mathrm{mM}$ nonessential amino acids (Invitrogen) and 0.1 $\mathrm{mg} / \mathrm{mL}$ streptomycin/100U penicillin solution (Invitrogen). Cells were cultivated in a humidified incubator with $5 \% \mathrm{CO}_{2}$ at $37^{\circ} \mathrm{C}$.

\section{Biofilm and planktonic cultures}

A. pleuropneumoniae was grown in 6-well plates (Costar® 3516, Corning, Corning, NY, USA) as previously described. ${ }^{30}$ After 4 hours in culture, media containing non-attached, planktonic bacteria were removed (the planktonic fraction). Attached bacteria were washed twice with phosphate buffered-saline (PBS) to remove remaining planktonic cells. Attached cells (the biofilm fraction) were scraped off the plate. Both planktonic and biofilm fractions were washed again with PBS, centrifuged and resuspended in fresh RPMI 1640 medium. Bacterial counts (CFU) and optical density were used to normalize bacterial concentrations. Bacteria were killed using UV irradiation (254 nm, $30 \mathrm{~min}$ ), then plated on BHI agar to verify the loss of viability. 


\section{Lipid A isolation}

Lipid A molecules were extracted from both planktonic and biofilm bacteria as described by El

Hamidi et al. ${ }^{31}$ Briefly, bacteria from both planktonic and biofilm fractions were lyophilized. Freeze-dried bacteria $(10 \mathrm{mg})$ were suspended in $200 \mu \mathrm{L}$ isobutyric acid-1M ammonium hydroxide mixture (5:3, v:v), and kept for $2 \mathrm{~h}$ at $100^{\circ} \mathrm{C}$ in a screw-cap test tube under agitation. Suspensions were cooled in ice, and then centrifuged $10 \mathrm{~min}$ at $2000 \mathrm{~g}$. Supernatants were diluted with 2 volumes of water and lyophilized. The dried materials were then washed twice with 200 $\mu \mathrm{L}$ of methanol. Finally, lipid A was extracted from pellets with $100 \mu \mathrm{L}$ of a mixture of chloroform, methanol and water (3:1.5:0.25, v:v:v).

\section{Mass spectrometry and data processing}

Analyses were performed using a Thermo Scientific Q-Exactive Orbitrap Mass Spectrometer (San Jose, CA, USA), interfaced with a Thermo Scientific UltiMate 3000 Rapid Separation UHPLC system, using a pneumatic assisted heated electrospray ion source (ESI). Chromatography was achieved using an isocratic mobile phase along with a microbore column Thermo Biobasic C8 $100 \times 1 \mathrm{~mm}$, with a particle size of $5 \mu \mathrm{m}$. The mobile phase consisted of methanol and water (fortified with $0.1 \%$ of formic acid) at a ratio of 95:5. The flow rate was fixed at $75 \mu \mathrm{L} / \mathrm{min}$ and $2 \mu \mathrm{L}$ samples were injected. Mass spectrometry was performed in positive ion mode and operated in scan mode at high-resolution and accurate-mass (HRAM). Nitrogen was used for sheath and auxiliary gases, set at 10 and 5 arbitrary units. The heated ESI probe was set to $4000 \mathrm{~V}$ and the ion transfer tube temperature was set to $300^{\circ} \mathrm{C}$. The scan range was set to m/z 1000-2500. Data were acquired at a resolving power of 140,000 (defined as full width at half maximum, FWHM), resulting in a scanning rate of $700 \mathrm{msec} / \mathrm{scan}$ when using 
automatic gain control target of $3.0 \times 10^{6}$ and maximum ion injection time of $200 \mathrm{msec}$. The instrument was calibrated prior to all analyses, and mass accuracy was notably below 1 ppm using Thermo Pierce calibration solution and automated instrument protocol. Differential analyses of planktonic versus biofilm were performed using Thermo Scientific SIEVE 2.1 software, which executes background subtraction, component detection, peak alignment and differential analyses. Parameters used in SIEVE were: time range 1-10 min, mass range 1000$2500 \mathrm{Da}$, frame width $10 \mathrm{mDa}$ and retention time width $2 \mathrm{~min}$. SIEVE output was transferred into SIEVE Extractor, an Excel spreadsheet and Lipid Maps databases. SIEVE software measures pvalues using a two-tailed Student's t-test. Fold changes and p-values were used to construct a volcano-plot and a Venn diagram analysis. Significance was set a priori to $p<0.01$ and only 2 fold changes were considered biologically significant.

\section{Stimulation of porcine pulmonary alveolar macrophages}

PAMs were prepared in 6-well plates (Costar $® 3516)$ to a final concentration of 1 x $10^{6}$ viable cells $/ \mathrm{ml}$ in $1.5 \mathrm{~mL}$ of RPMI 1640 medium. Plates were incubated at $37^{\circ} \mathrm{C}$ in a humidified incubator $5 \% \mathrm{CO}_{2}$ for $24 \mathrm{~h}$. Medium was removed and fresh medium containing planktonic or biofilm bacteria in suspension with a multiplicity of infection (MOI) of 10:1 was added to the wells $^{32}$. As negative control, medium alone was added. Exposed and non-exposed cells were incubated for 1 to $24 \mathrm{~h}$ at $37^{\circ} \mathrm{C}, 5 \% \mathrm{CO}_{2}$.

\section{Cytokine mRNA expression}

The expression of several pro-inflammatory cytokines was determined in PAMs exposed to planktonic or biofilm bacteria using qRT-PCR. To begin, non-adherent PAMs were rinsed away, and the remaining attached macrophages were collected in Trizol reagent, and RNA was 
extracted as described by the manufacturer (Invitrogen). Purified mRNA was resuspended in $30 \mu \mathrm{L}$ of RNase-free water. Total RNA from samples was stored at $-80^{\circ} \mathrm{C}$ until reversetranscription was done. Quantification of RNA was performed with a Nanodrop (NanoDrop Technologies, Inc., Wilmington, DE, USA). $1 \mu \mathrm{g}$ of total RNA was reverse-transcribed using the QuantiTect reverse transcription kit (Qiagen, Mississauga, ON, Canada) according to manufacturer's instructions. cDNA was amplified using the SsoFast ${ }^{\mathrm{TM}}$ EvaGreen_Supermix kit (Bio-rad, Hercules, CA, USA). The primers used for amplification ${ }^{32-34}$ are presented in Table $\mathbf{1 .}$ Primer sequences were all designed from the NCBI GenBank mRNA sequences using web-based software primerquest from Integrated DNA technologies (www.idtdna.com/SciTools). 16-place

Cepheid Smart Cycler $^{\circledR}$ System apparatus (Cepheid, Sunnyvale, CA, USA), was used for the cDNA amplification. Quantification of differences between groups was calculated using the $2^{-}$ $\Delta \Delta \mathrm{Ct}$ method. $\beta 2$-microglobulin was used as normalizing gene to compensate for potential differences in cDNA amounts. Non-infected PAM cells were used as a calibrator in the analysis.

\section{IL-8 quantification by ELISA}

Levels of IL-8 in cell culture supernatants were measured by sandwich ELISA using pairmatched antibodies from R\&D Systems (Minneapolis, MN, USA), according to the manufacturer's recommendations. Twofold dilutions of recombinant porcine IL-8 (R\&D Systems) were used to generate the standard curves. Sample dilutions giving optical density readings in the linear portion of the standard curve were used to quantify the levels of IL-8. 


\section{Proliferation of porcine peripheral blood mononuclear cells}

The procedures for animal care followed the guidelines of the Canadian Council on Animal Care and the protocol for blood collection was approved by our Institutional Animal Care Committee (Protocol 13-Rech-1558). Blood was collected from 4 healthy pigs (3 to 10 weeks of age) into heparinized tubes, and mononuclear cells were isolated using lymphocyte separation medium (Wisent, St-Bruno, QC, Canada) centrifugation at $400 \times \mathrm{g}$ for $30 \mathrm{~min}$. The buffy coat containing mononuclear cells was isolated, transferred to a fresh centrifuge tube, and washed twice with PBS. The final cell pellet containing peripheral blood mononuclear cells (PBMC) was resuspended to a final level of $2 \times 10^{6}$ cells/mL in RPMI 1640 medium supplemented with 25 mM HEPES buffer, $2 \mathrm{mM}$ L-glutamine, $1 \mathrm{mM}$ sodium pyruvate, $5 \times 10^{-5} \mathrm{M}$ mercaptoethanol (Invitrogen), $0.1 \mathrm{mg} / \mathrm{mL}$ streptomycin/100U penicillin solution and 10\% FBS (heat inactivated). Fresh isolated PBMCs cells were incubated with planktonic and biofilm cells $\left(2 \times 10^{7}\right.$ cells $\left./ \mathrm{mL}\right)$. As positive control, concanavalin A (5 ug/mL; Sigma-Aldrich, St-Louis, MO, USA) was used and fresh RPMI 1640 medium was used as negative control.

Cell proliferation was measured upon completion of the $48 \mathrm{~h}$ incubation period using a BrdUELISA kit (Millipore, Billerica, MA, USA) as per manufacturer instructions. In brief, kitprovided BrdU labeling solution $(20 \mu \mathrm{L})$ was added to each well and the plate was incubated at $37^{\circ} \mathrm{C}$ for $24 \mathrm{~h}$. Thereafter, the cells were centrifuged at $300 \times \mathrm{g}$ for $10 \mathrm{~min}$, the labeling solution was removed, and the cells were fixed by addition of fixative/denaturing solution ( $200 \mu \mathrm{L} /$ well) and incubation at room temperature for $30 \mathrm{~min}$. Antibody conjugate (anti-BrdU solution, 100 $\mu \mathrm{L} /$ well) was then added and the plate was incubated at room temperature for $1 \mathrm{~h}$. The cells were then washed twice with washing buffer $(200 \mu \mathrm{L})$ and a peroxidase goat anti-mouse IgG HRP conjugate $(100 \mu \mathrm{L})$ was added to each well for 30 min. Again, the cells were washed twice with 
washing buffer $(200 \mu \mathrm{L})$. Substrate solution $(100 \mu \mathrm{L})$ was added to the wells and incubated in the dark at room temperature for $15 \mathrm{~min}$. Then $100 \mu \mathrm{L}$ of stop solution was added and the absorbance in each well was measured at $450 \mathrm{~nm}$ in an automated plate reader (Powerwave, BioTek Instruments, Winooski, VT, USA). Blanks (100 $\mu \mathrm{L}$ culture medium alone) and control wells were included in each experiment.

\section{Statistical analysis}

qRT-PCR, ELISA and PBMCs proliferation assays data were analyzed statistically using GraphPad Prism software (version 4.03, GraphPad Software Inc., San Diego, CA) by application of Wilcoxon-Mann-Whitney test or one-way ANOVA with Bartlett's test. $p<0.05$ or $<0.01$ were considered reflecting statistically significant differences. 


\section{RESULTS}

\section{Lipid A structure analysis}

Lipid A molecules were extracted following a validated protocol ${ }^{31}$ and their relative abundance in A. pleuropneumoniae planktonic and biofilm cells were analyzed using mass spectrometry. For both planktonic and biofilm cells, a total of 228 distinct molecular entities were observed and compared. Analyses were performed using an untargeted HPLC-HRAM MS lipidomic workflow. After data acquisition, the results were processed using bioinformatics software SIEVE 2.1 to perform retention time alignment and identify specific ions (i.e $\mathrm{m} / \mathrm{z}$ ) that differed between planktonic and biofilm cell groups. The $\mathrm{m} / \mathrm{z}$ values for peaks of interest were searched in public metabolite/lipid databases (i.e. METLIN, mzCloud and Lipid MAPS) to obtain putative identifications without success. This is not surprising since the lipid A molecular structure and associated molecules for A. pleuropneumoniae remain unknown and not available in public databases. Following peak alignment and identification from biofilm lipid A (n = 3) and planktonic lipid A ( $\mathrm{n}=3)$, relative ion intensities and $\mathrm{m} / \mathrm{z}$ values were used to study the molecular abundance distribution and modifications between the two cell states. Extracted data were represented using volcano plot with a $p$ value $=0.01(\mathrm{CI}$ of 99\%) and a two-fold change cut off were assumed to be biologically significant. Figure 1(a) illustrates the significant variations in lipid A abundance and molecular structures between A. pleuropneumoniae planktonic and biofilm cells with representative chromatograms shown in Figure 1(b).

Following an exhaustive analysis, a total of 23 lipid A structures in the planktonic fraction and 17 in the biofilm fraction were found to be significantly different (Figure 2(a)). Inspection of the 
detected peaks $(\mathrm{m} / \mathrm{z})$ revealed an interesting shift of the lipid A towards higher molecular weight in biofilm cells, as shown in Figure 2(b).

\section{Activation of Macrophages}

Relative mRNA expression of pro-inflammatory cytokine genes (IL-1, IL-6, IL-8 and MCP-1) was measured in PAM cells following exposition to either inactivated A. pleuropneumoniae biofilm or planktonic cells. The expression of all tested cytokines mRNA was up-regulated when PAMs were exposed to A. pleuropneumoniae cells compared to unexposed control cells. qRTPCR data showed an early expression of all cytokines tested when exposed to planktonic cells (Figure 3). A peak value is reached at $1 \mathrm{~h}$ post-stimulation and declined shortly except for IL-8, which demonstrated a particularly high and sustained expression compared with the other cytokines. Two other peaks at $8 \mathrm{~h}$ and $24 \mathrm{~h}$ post-stimulation for the MCP-1, and one peak for the IL-1 at $24 \mathrm{~h}$ post-stimulation were observed. These peaks could be the result of an autoactivation process caused by the increase of cytokines in the culture media.

Interestingly, exposition of macrophages to A. pleuropneumoniae biofilm cells appeared to induce significantly lower expression of pro-inflammatory cytokines and chemokines in general compared to macrophages exposed to planktonic cells $(P<0.05)$ (Figure 3).

The level of cytokine IL-8 in the supernatant of PAM cells following exposition to either $A$. pleuropneumoniae biofilm or planktonic cells was also measured by ELISA at $1 \mathrm{~h}, 2 \mathrm{~h}, 4 \mathrm{~h}$ and $8 \mathrm{~h}$ (Figure 4). Our results demonstrated that the amount of IL-8 tends to increase with time. As expected, levels of IL-8 detected at $4 \mathrm{~h}$ and $8 \mathrm{~h}$ post-stimulation with biofilm cells were significantly lower compared to levels obtained with planktonic cells $(P<0.05)$, confirming mRNA results. 


\section{Porcine peripheral blood mononuclear cells proliferation}

Since A. pleuropneumoniae biofilm induces less activation of PAMs, we hypothesized that biofilms cells could also modulate the proliferative response of PBMCs. PBMCs were exposed to both biofilm and planktonic cells, and the proliferation was evaluated and compared to unexposed cells (negative controls) (Figure 5). As expected, a significantly higher proliferation was observed when immune cells were exposed to concanavalin A or A. pleuropneumoniae cells (planktonic and biofilm) compared to unexposed cells control $(P<0.05)$. The proliferative response of PBMCs exposed to planktonic and biofilm cells was then evaluated. Biofilm cells induced a lower response compared to their planktonic counterparts $(P<0.05)$ (Figure 5). 


\section{DISCUSSION}

Lipopolysaccharide (LPS) has been implicated as one of the major virulence factors in $A$. pleuropneumoniae $e^{18,36-38}$ and other Gram-negative bacteria. ${ }^{39,40}$ LPS has several functions. First of all, it protects bacteria by establishing a permeability barrier. ${ }^{40-42}$ The biological activity of LPS stems from its hydrophobic anchor, lipid $\mathrm{A}^{39}$ which stimulates the Toll-like receptor 4 (TLR4), found on the surface of monocytes, macrophages and neutrophils. ${ }^{43-45}$ Lipid A will first bind the co-receptor MD-2, before interacting with the TLR4 receptor. ${ }^{43,44}$ The formed complex TLR-4/MD-2/LPS subsequently activates transcription factor NF-kB and pro-inflammatory cytokines IL-1, IL-6 and IL-8. ${ }^{45,46}$ Several structural modifications in lipid A, such as the loss of phosphate groups and variation in length and number of the fatty acyl chains, have an important role in TLR4 recognition and response. ${ }^{40,45,47-50}$ Variations in lipid A structure provide bacteria fitness advantages to colonize surfaces, evade the immune system and persist during infections. ${ }^{49,51,52}$ Some of these variations are associated with chronic infection ${ }^{51,53}$ and biofilm formation. $^{47}$

Biofilm bacteria are encased within a polymeric matrix that contains among other things, polysaccharides, extracellular DNA, proteins and lipids. ${ }^{54-56}$ They are frequently associated with chronic disease $\mathrm{e}^{25,26,57}$ and represent a serious therapeutic challenge, since bacteria within biofilm exhibit enhanced antibiotic tolerance and are more resistant to clearance by the immune system. ${ }^{3,11,24,57}$ We recently demonstrated that LPS has an important role in biofilm formation in A. pleuropneumoniae, and that a biofilm matrix exopolysaccharide, poly-N-acetyl-Dglucosamine, can interact specifically with LPS ${ }^{30}$. However, the impact on host cells of LPS and particularly lipid A in biofilms remains to be determined. 
In this study, using an HPLC-HRAM MS untargeted lipidomic based approach, we demonstrated that lipid A molecular structure seems to be influenced by the bacterial mode of growth (sessile or planktonic). Planktonic and biofilm cells differed significantly in lipid A structure and quantity, with larger lipid A molecular entities observed in biofilm cells. This constitutes an additional manner biofilm cells differ phenotypically from their free-floating counterparts. ${ }^{58-60}$ However, further analyses will be required to determine molecular identity (i.e. extensive purification and ${ }^{1} \mathrm{H} /{ }^{13} \mathrm{C}$ NMR).

Lipid A contributes to cell rigidity by increasing cell wall strength. ${ }^{61,62}$ During biofilm formation, embedded bacteria may exert pressure on their neighbors, ${ }^{62}$ and the increased molecular interactions could favor reactions leading to modified lipid A structures and cell surface remodeling.

Several studies have demonstrated that biofilms can undergo LPS structural modifications that modulate the immune response. ${ }^{47,48,51}$ Recently, Chalabaev and colleagues described an interesting biofilm associated-phenotype linked to lipid A structure. ${ }^{47}$ They found that among several Gram-negative bacteria, including Escherichia coli and Pseudomonas aeruginosa, lipid A can undergo palmitoylation, and these changes were found to be more abundant in biofilm than in planktonic cells. ${ }^{47}$ Lipid A structural modifications, including addition of arabinose and palmitate were also observed in $P$. aeruginosa strains isolated from patients with cystic fibrosis, a chronic lung infection wherein biofilms have a preponderant role. ${ }^{51,53}$ These modifications were associated with resistance to host innate defenses and persistence. ${ }^{53}$ In biofilms, lipid A modification may confer a fitness advantage by enabling bacteria to escape and survive host defense systems, by increasing steric hindrance, and by promoting chronic infection. 
Cytokines like IL-1, IL-6, IL-8 and MCP-1 play important roles in mediating inflammatory response to pathogens. Pro-inflammatory IL-8 and MCP-1 trigger neutrophils migration to the site of the infection. One of our objectives was to see how planktonic and biofilm $A$. pleuropneumoniae cells interact with immune cells. Our data indicate that biofilm cells trigger a different innate immune response than do planktonic cells. Previous studies showed that $A$. pleuropneumoniae infections were followed by increased TNF, IL-1 and IL-8 mRNA expression. These cytokines were detected at the periphery of the lung lesion. ${ }^{17,63}$ Increased inflammatory cytokine levels were also seen in lung lesions from acute porcine pleuropneumonia. ${ }^{17,63}$

Using IL-10 gene therapy, Morrison et al. demonstrated that this anti-inflammatory cytokine can diminish levels of pro-inflammatory cytokines, IL-1 and TNF $\alpha$, and consequently reduce lung damage and severity in pleuropneumonia. ${ }^{20}$ In this study, we observed that levels of proinflammatory cytokines were significantly lower in PAMs exposed to biofilm cells compared to those exposed to planktonic cells. Furthermore, porcine PBMCs exhibited a lower proliferative response when exposed to biofilm compared to planktonic cells. Overall our data suggest that the biofilm mode of growth mitigates the innate immune response to this pathogen.

The role of biofilm in chronic disease and low-level inflammation response is increasingly recognized. Many studies support this hypothesis. ${ }^{64,65}$ Daw et al. showed that macrophages infected with of Enterococcus faecalis biofilm cells secreted lower pro-inflammatory cytokine levels compared to planktonic cells. ${ }^{66}$ Similarly, macrophages infected with Legionella pneumophila biofilm exhibited significantly lower levels of pro-inflammatory cytokine IL-1 $\beta$, compared to those infected with planktonic bacteria. ${ }^{67}$ In this way, biofilm-derived L. pneumophila avoids major inflammatory responses and recruitment of inflammatory cells. ${ }^{67}$ Similarly, Thurlow et al. demonstrated that Staphylococcus aureus biofilm evades TLR recognition and alters the immune response to infection. ${ }^{68}$ Indeed, several inflammatory signals 
including MCP-1, TNF- $\alpha$, and IL-1 $\beta$ were significantly attenuated in $S$. aureus biofilm-infected tissues compared to the wound healing response elicited by insertion of sterile catheter. ${ }^{68}$

Less lung lesions and low inflammation and are often associated with the chronic form of pleuropneumonia $^{17,20,63}$ suggesting the involvement of biofilm. A. pleuropneumoniae growing in biofilm may overcome host defenses by modifying its lipid A structure, which enables a lower immune reactivity. However, lipid A structural modifications may not be the sole cause of the immune dysfunction observed here and other bacterial surface components may also be implicated. Further studies will be needed to demonstrate the direct impact of these biofilm associated-lipid A structural modifications in the altered immune response.

The lipid A structure in A. pleuropneumoniae has yet to be elucidated. However, fatty acids in the lipid A of A. pleuropneumoniae serotype 1 were identified, and it was shown that lipid A contains different proportions of n-dodecanoic acid (0.6\%), n-tetradecanoic acid (12.9\%), 3hydroxytetradecanoic acid (64.3\%), n-hexadecanoic acid (3.2\%), and n-octadecanoic acid $(0.6 \%) .{ }^{69}$ Knowing lipid A structure and the fatty acids arrangement in A. pleuropneumoniae would help to identify the modifications occurring in lipid A during the switch from planktonic to biofilm form and identify their impact on immune response.

This study demonstrates that the A. pleuropneumoniae biofilm mode of growth has an impact on lipid A structure and mitigates the innate immune response. Further investigation must focus on these lipid A modifications associated with lower immune cells stimulation. 


\section{ACKNOWLEDGEMENTS}

The mutant strain A. pleuropneumoniae MBHPP147 was kindly provided by Ruud P.A.M Segers (MSD Animal Health, Boxmeer, The Netherlands). We would like to thanks Philip Dominico for reading the manuscript and providing precious advices. Thermo Fisher Scientific provided generous access to a Q-Exactive Orbitrap Mass Spectrometer to FB.

\section{FUNDING}

This work was supported by a Discovery grant from the Natural Sciences and Engineering Research Council of Canada (NSERC grant 003428) to MJ; SH and LM received a scholarship from the Centre de recherche en infectiologie porcine et avicole (CRIPA); the analyses were performed using analytical instruments acquired with an NSERC Research Tools and Instruments grant to FB (439748-2013); partial funding was also provided by NSERC (grant 342150) to MS.

\section{CONFLICT OF INTEREST}

The authors declare that they have no conflicts of interest with the contents of this article. 


\section{REFERENCES}

1. Costerton JW. Introduction to biofilm. Int J Antimicrob Agents 1999; 11:217-221.

2. Hall-Stoodley L, Costerton JW and Stoodley P. Bacterial biofilms: from the natural environment to infectious diseases. Nat Rev Microbiol 2004; 2:95-108.

3. Hoiby N, Bjarnsholt T, Givskov M et al. Antibiotic resistance of bacterial biofilms. Int J Antimicrob Agents 2010; 35:322-332.

4. Donlan RM and Costerton JW. Biofilms: survival mechanisms of clinically relevant microorganisms. Clin Microbiol Rev 2002; 15:167-193.

5. Cerca F, Franca A, Perez-Cabezas B et al. Dormant bacteria within Staphylococcus epidermidis biofilms have low inflammatory properties and maintain tolerance to vancomycin and penicillin after entering planktonic growth. J Med Microbiol 2014; doi:10.1099/jmm.0.073163-0.

6. Ciofu O, Tolker-Nielsen T, Jensen PO et al., Antimicrobial resistance, respiratory tract infections and role of biofilms in lung infections in cystic fibrosis patients. Adv Drug Deliv Rev 2014 ; doi:10.1016/j.addr.2014.11.017.

7. Kaplan $\mathbf{J}$ and Mulks M. Biofilm formation is prevalent among field isolates of Actinobacillus pleuropneumoniae. Vet Microbiol 2005; 108:89 - 94.

8. Tremblay Y, Deslandes V and Jacques M. Actinobacillus pleuropneumoniae genes expression in biofilms cultured under static conditions and in a drip-flow apparatus. $B M C$ Genomics 2013; 14:364.

9. Labrie J, Pelletier-Jacques G, Deslandes V et al. Effects of growth conditions on biofilm formation by Actinobacillus pleuropneumoniae. Vet Res 2010 ; 41:3. 
10. Tremblay YD, Lévesque C, Segers RP et al. Method to grow Actinobacillus pleuropneumoniae biofilm on a biotic surface. BMC Vet Res 2013; 9:213.

11. Archambault M, Harel J, Goure J et al. Antimicrobial susceptibilities and resistance genes of Canadian isolates of Actinobacillus pleuropneumoniae. Microb Drug Res 2012; 18:198 - 206.

12. Losinger WC. Economic impacts of reduced pork production associated with the diagnosis of Actinobacillus pleuropneumoniae on grower/finisher swine operations in the United States. Prev Vet Med 2005; 68:181-193.

13. Bosse J, Janson H, Sheehan B et al. Actinobacillus pleuropneumoniae: pathobiology and pathogenesis of infection. Microbes Infect 2002; 4:225 - 235.

14. Gottschalk M. Actinobacillosis. In: L Karriker, A Ramirez, K Schwartz, G Stevenson, J Zimmerman (eds). Diseases of Swine. 10th ed. Wiley: Hoboken, NJ, 2012, pp. 653-669.

15. Rayamajhi N, Shin SJ, Kang SG et al. Development and use of a multiplex polymerase chain reaction assay based on Apx toxin genes for genotyping of Actinobacillus pleuropneumoniae isolates. J Vet Diagn Invest 2005; 17:359-362.

16. Haesebrouck F, Chiers K, Van Overbeke I et al. Actinobacillus pleuropneumoniae infections in pigs: the role of virulence factors in pathogenesis and protection. Vet Microbiol 1997; 58:239-249.

17. Baarsch MJ, Scamurra RW, Burger K et al. Inflammatory cytokine expression in swine experimentally infected with Actinobacillus pleuropneumoniae. Infect Immun 1995; 63:3587-3594.

18. Ramjeet M, Deslandes V, St Michael F et al. Truncation of the lipopolysaccharide outer core affects susceptibility to antimicrobial peptides and virulence of Actinobacillus pleuropneumoniae serotype 1. J Biol Chem 2005; 280:39104-39114. 
19. Huang $\mathrm{H}$, Potter AA, Campos $\mathrm{M}$ et al. Pathogenesis of porcine Actinobacillus pleuropneumonia, part II: roles of proinflammatory cytokines. Can J Vet Res 1999; 63:69-78.

20. Morrison DF, Foss DL and Murtaugh MP. Interleukin-10 gene therapy-mediated amelioration of bacterial pneumonia. Infect Immun 2000; 68:4752-4758.

21. Reiner G, Fresen C, Bronnert S et al. Prevalence of Actinobacillus pleuropneumoniae infection in hunted wild boars (Sus scrofa) in Germany. $J$ Wildl Dis 2010; 46:551-555.

22. MacInnes JI, Gottschalk M, Lone AG, et al. Prevalence of Actinobacillus pleuropneumoniae, Actinobacillus suis, Haemophilus parasuis, Pasteurella multocida, and Streptococcus suis in representative Ontario swine herds. Can J Vet Res 2008; 72:242-248.

23. Alhede $\mathrm{M}$, Bjarnsholt $\mathrm{T}$, Givskov $\mathrm{M}$ et al. Pseudomonas aeruginosa biofilms: mechanisms of immune evasion. Adv Appl Microbiol 2014; 86:1-40.

24. Domenech M, Ramos-Sevillano E, Garcia E et al. Biofilm formation avoids complement immunity and phagocytosis of Streptococcus pneumoniae. Infect Immun 2013 ; 81:26062615.

25. Vuong C, Kocianova S, Voyich JM et al. A crucial role for exopolysaccharide modification in bacterial biofilm formation, immune evasion, and virulence. J Biol Chem 2004; 279:54881-54886.

26. Bjarnsholt T.. The role of bacterial biofilms in chronic infections. APMIS Suppl 2013; doi:10.1111/apm.12099:1-51.

27. Burmolle M, Thomsen TR, Fazli M et al. Biofilms in chronic infections - a matter of opportunity - monospecies biofilms in multispecies infections. FEMS Immunol Med Microbiol 2010; 59:324-336. 
28. Jacques M, Aragon V and Tremblay Y. Biofilm formation in bacterial pathogens of veterinary importance. Anim Health Res Rev 2010; 11:97 - 121.

29. Clutterbuck AL, Woods EJ, Knottenbelt DC et al. Biofilms and their relevance to veterinary medicine. Vet Microbiol 2007; 121:1-17.

30. Hathroubi S, Hancock MA, Bosse JT et al. Surface polysaccharide mutants reveal that absence of $\mathrm{O}$ antigen reduces biofilm formation of Actinobacillus pleuropneumoniae. Infect Immun 2015; 84:127-137 doi:10.1128/IAI.00912-15.

31. El Hamidi A, Tirsoaga A, Novikov A et al. Microextraction of bacterial lipid A: easy and rapid method for mass spectrometric characterization. J Lipid Res 2005; 46:1773-1778.

32. Lévesque C, Provost C, Labrie J et al. Actinobacillus pleuropneumoniae possesses an antiviral activity against porcine reproductive and respiratory syndrome virus. PLoS One 2014 9: e98434.

33. Savard C, Pinilla V, Provost C et al. In vitro effect of deoxynivalenol (DON) mycotoxin on porcine reproductive and respiratory syndrome virus replication. Food Chem Toxicol 2014; 65:219-226.

34. Dang $\mathrm{Y}$, Lachance $\mathrm{C}$, Wang $\mathrm{Y}$ et al. Transcriptional approach to study porcine tracheal epithelial cells individually or dually infected with swine influenza virus and Streptococcus suis. BMC Vet Res 2014; 10:86.

35. Wang Y, Gagnon CA, Savard C et al. Capsular sialic acid of Streptococcus suis serotype 2 binds to swine influenza virus and enhances bacterial interactions with virus-infected tracheal epithelial cells. Infect Immun 2013; 81:4498-4508. 
36. Jacques M. Surface polysaccharides and iron-uptake systems of Actinobacillus pleuropneumoniae. Can J Vet Res 2004; 68:81-85.

37. Ramjeet M, Cox AD, Hancock MA et al. Mutation in the LPS outer core biosynthesis gene, galU, affects LPS interaction with the RTX toxins ApxI and ApxII and cytolytic activity of Actinobacillus pleuropneumoniae serotype 1. Mol Microbiol 2008; 70:221235.

38. Huang H, Potter AA, Campos M, et al. Pathogenesis of porcine Actinobacillus pleuropneumonia: Part I. Effects of surface components of Actinobacillus pleuropneumoniae in vitro and in vivo. Can J Vet Res 1998; 62:93-101.

39. Raetz CR and Whitfield C. Lipopolysaccharide endotoxins. Annu Rev Biochem 2002, 71:635-700.

40. Penterman J, Nguyen D, Anderson E et al. Rapid evolution of culture-impaired bacteria during adaptation to biofilm growth. Cell Rep 2014 ; 6:293-300.

41. Snyder DS and McIntosh TJ. The lipopolysaccharide barrier: correlation of antibiotic susceptibility with antibiotic permeability and fluorescent probe binding kinetics. Biochemistry 2000, 39:11777-11787.

42. Vaara M and Nurminen M. 1999. Outer membrane permeability barrier in Escherichia coli mutants that are defective in the late acyltransferases of lipid A biosynthesis. Antimicrob Agents Chemother 43:1459-1462.

43. Meng J, Gong M, Björkbacka $\mathrm{H}$ et al. Genome-wide expression profiling and mutagenesis studies reveal that ipopolysaccharide responsiveness appears to be absolutely dependent on TLR4 and MD-2 expression and is dependent upon intermolecular ionic interactions. $J$ Immunol 2011; 187:3683-3693. 
44. Park BS and Lee JO. Recognition of lipopolysaccharide pattern by TLR4 complexes. Exp Mol Med 2013; 45:e66.

45. Shah NR, Albitar-Nehme S, Kim E, et al. Minor modifications to the phosphate groups and the C3' acyl chain length of lipid A in two Bordetella pertussis strains, BP338 and 18323, independently affect Toll-like receptor 4 protein activation. J Biol Chem 2013; 288:11751-11760.

46. Medzhitov R and Janeway C, Jr. Innate immune recognition: mechanisms and pathways. Immunol Rev 2000; 173:89-97.

47. Chalabaev S, Chauhan A, Novikov A et al. Biofilms formed by gram-negative bacteria undergo increased lipid a palmitoylation, enhancing in vivo survival. MBio 2014; 5.

48. Ciornei CD, Novikov A, Beloin C et al. Biofilm-forming Pseudomonas aeruginosa bacteria undergo lipopolysaccharide structural modifications and induce enhanced inflammatory cytokine response in human monocytes. Innate Immun 2010; 16:288-301.

49. Needham BD and Trent MS. Fortifying the barrier: the impact of lipid A remodelling on bacterial pathogenesis. Nat Rev Microbiol 2013; 11:467-481.

50. Raetz CR, Reynolds CM, Trent MS et al. Lipid A modification systems in gram-negative bacteria. Аппи Rev Biochem 2007; 76:295-329.

51. Ernst RK, Moskowitz SM, Emerson JC et al. Unique lipid a modifications in Pseudomonas aeruginosa isolated from the airways of patients with cystic fibrosis. $J$ Infect Dis 2007; 196:1088-1092.

52. Kawahara $\mathrm{K}$, Tsukano $\mathrm{H}$, Watanabe $\mathrm{H}$ et al. Modification of the structure and activity of lipid A in Yersinia pestis lipopolysaccharide by growth temperature. Infect Immun 2002; 70:4092-4098. 
53. Ernst RK, Adams KN, Moskowitz SM et al.. The Pseudomonas aeruginosa lipid A deacylase: selection for expression and loss within the cystic fibrosis airway. $J$ Bacteriol 2006; 188:191-201.

54. Öner ET. Microbial Production of Extracellular Polysaccharides from Biomass, p 35-56. In: Fang Z (ed) Pretreatment Techniques for Biofuels and Biorefineries. Springer Berlin Heidelberg, 2013, doi:10.1007/978-3-642-32735-3_2.

55. Flemming HC and Wingender J. The biofilm matrix. Nat Rev Microbiol 2010; 8:623-633.

56. Flemming HC, Neu TR and Wozniak DJ. The EPS matrix: the "house of biofilm cells". $J$ Bacteriol 2007; 189:7945-7947.

57. Costerton J, Stewart P and Greenberg E. Bacterial biofilms: a common cause of persistent infections. Science 1999; 284:1318 - 1322.

58. Sauer K, Camper AK, Ehrlich GD, et al. Pseudomonas aeruginosa displays multiple phenotypes during development as a biofilm. J Bacteriol 2002; 184:1140-1154.

59. Drenkard E and Ausubel FM. Pseudomonas biofilm formation and antibiotic resistance are linked to phenotypic variation. Nature 2002; 416:740-743.

60. Stewart PS and Franklin MJ. Physiological heterogeneity in biofilms. Nat Rev Microbiol 2008; 6:199-210.

61. Silhavy TJ, Kahne D and Walker S. The bacterial cell envelope. Cold Spring Harb Perspect Biol 2010; 2:a000414.

62. Lambert G, Bergman A, Zhang QC et al. Physics of biofilms: the initial stages of biofilm formation and dynamics. New J Phys 2014; 16.

63. Brogaard L, Klitgaard K, Heegaard PM et al. Concurrent host-pathogen gene expression in the lungs of pigs challenged with Actinobacillus pleuropneumoniae. BMC Genomics 2015; 16:417. 
64. Roilides E, Simitsopoulou M, Katragkou A et al. How biofilms evade host defenses. Microbiol Spectr 2015 ; doi: 10.1128/microbiolspec.

65. Hanke ML and Kielian T. Deciphering mechanisms of staphylococcal biofilm evasion of host immunity. Front Cell Infect Microbiol 2012; 2:62.

66. Daw K, Baghdayan AS, Awasthi S et al. Biofilm and planktonic Enterococcus faecalis elicit different responses from host phagocytes in vitro. FEMS Immunol Med Microbiol 2012; 65:270-282.

67. Abu Khweek A, Fernandez Davila NS, Caution K et al. Biofilm-derived Legionella pneumophila evades the innate immune response in macrophages. Front Cell Infect Microbiol 2013; 3:18.

68. Thurlow LR, Hanke ML, Fritz T et al. Staphylococcus aureus biofilms prevent macrophage phagocytosis and attenuate inflammation in vivo. J Immunol 2011; 186:6585-6596.

69. Altman E, Brisson JR and Perry MB. Structure of O-chain of the lipopolysaccharides of Haemophilus pleuropneumoniae seroptype 1. Biochem Cell Biol 1986; 64:1317-1325 
Table 1. Primers used for quantitative real-time reverse-transcriptase (RT)-PCR.

\begin{tabular}{|c|c|c|c|}
\hline Primer & Sequence & Size (bp) & References \\
\hline \multirow[t]{2}{*}{$\beta 2 \mathrm{~m}$} & B2m_Fw CGTGGCCTTGGTCCTGCTCG & 103 & (33) \\
\hline & B2m_Rev TCCGTTTTCCGCTGGGTGGC & & \\
\hline \multirow[t]{2}{*}{ IL-1a } & IL1a_Fw TGAAGATGGCCAAAGTCCCTGACCT & 152 & $(34)$ \\
\hline & IL1a_Rev ATCCATGCCGTCCCCAGGAAGTG & & \\
\hline \multirow[t]{2}{*}{ IL-6 } & IL6_Fw ACTCCCTCTCCACAAGCGCCTT & 105 & (34) \\
\hline & IL6_Rev TGGCATCTTCTTCCAGGCGTCCC & & \\
\hline \multirow[t]{2}{*}{ IL-8 } & IL8_Fw TGTGAGGCTGCAGTTCTGGCAAG & 80 & (34) \\
\hline & IL8_Rev GGGTGGAAAGGTGTGGAATGCGT & & \\
\hline \multirow[t]{2}{*}{ MCP-1 } & MCP-1_Fw CAGGTCCTTGCCCAGCCAGATG & 143 & $(35)$ \\
\hline & MCP-1_Rev CACAGATCTCCTTGCCCGCGA & & \\
\hline
\end{tabular}




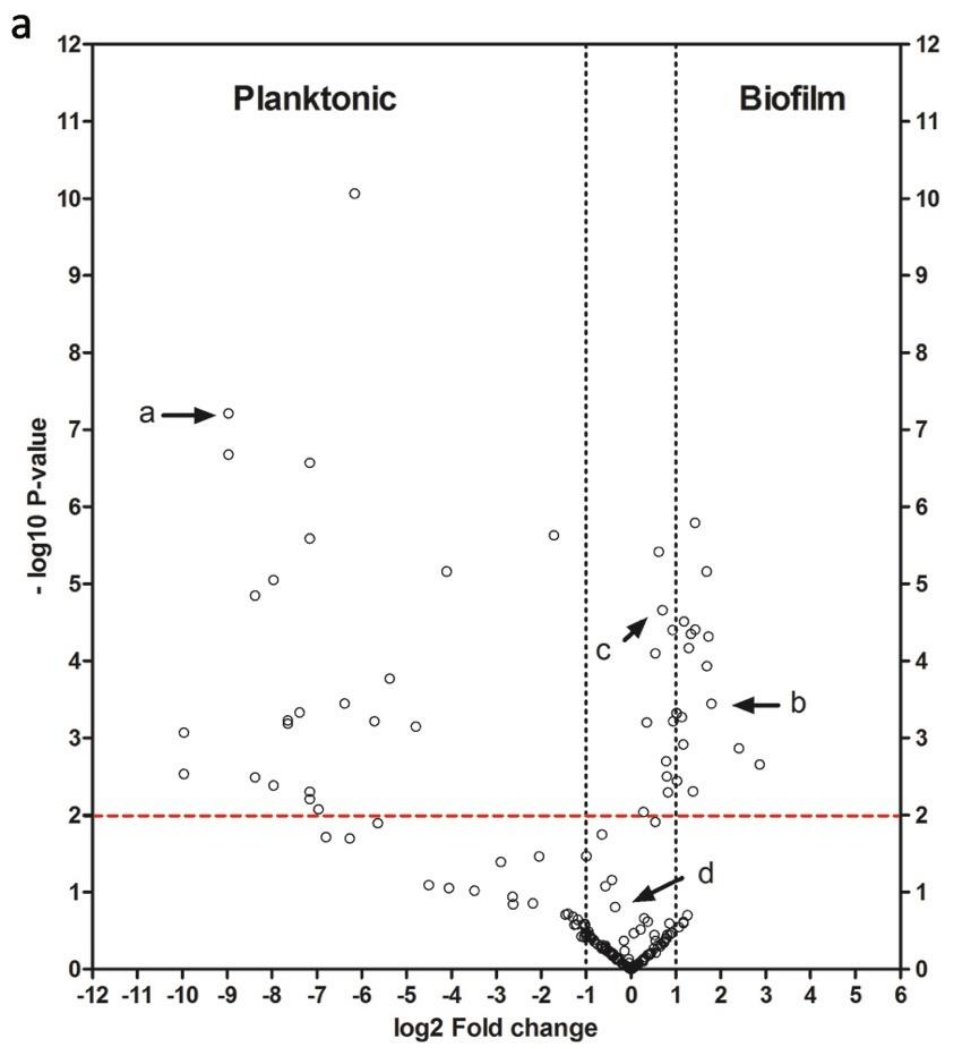

b
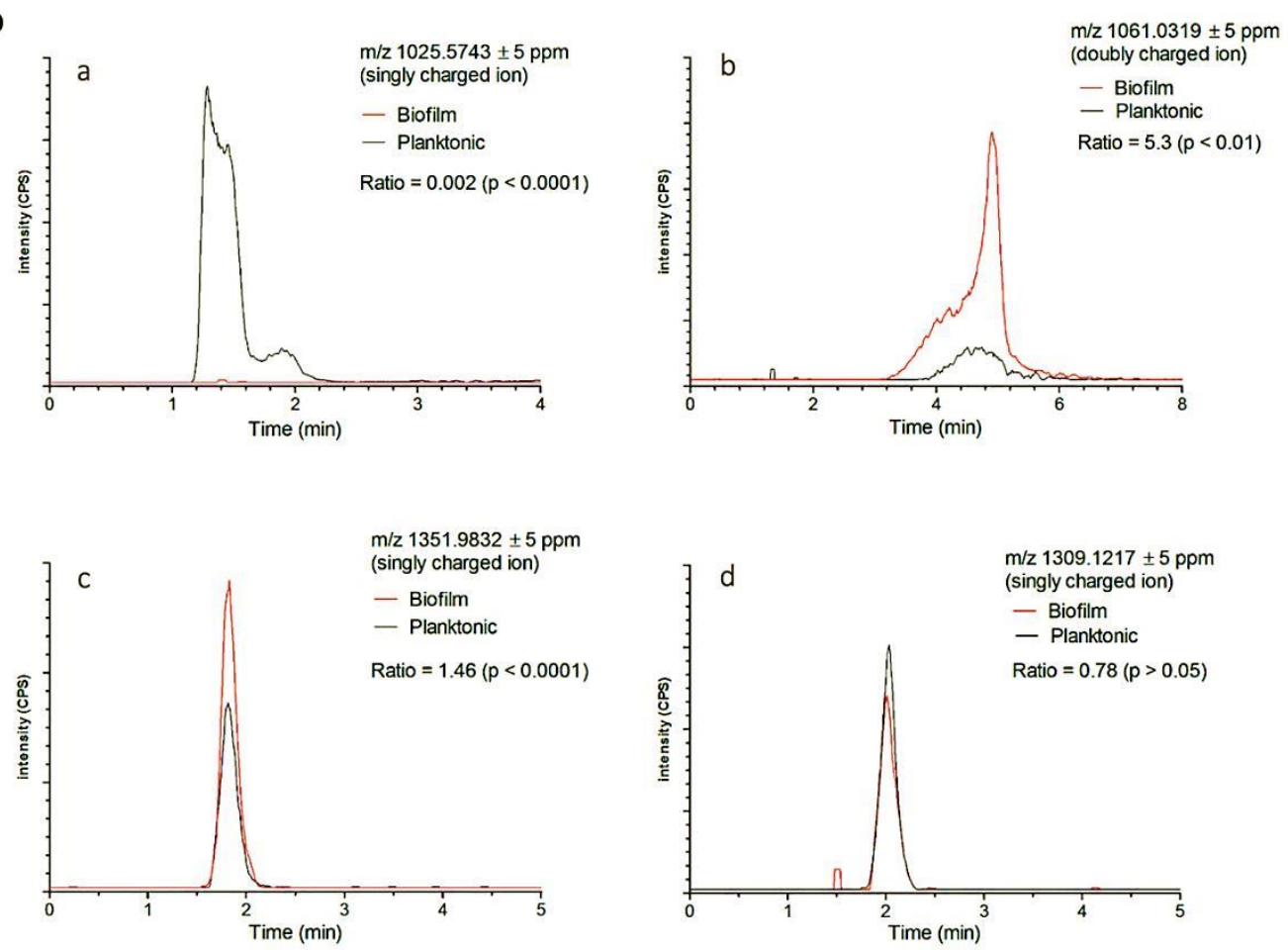
Figure 1. (a) Volcano plot of distribution of A. pleuropneumoniae lipid A structures isolated from biofilm cells $(n=3)$ or planktonic cells $(n=3)$. The volcano plot displays the relationship between fold-change and significance between the two groups, using a scatter plot view. The yaxis is the negative $\log 10$ of $P$ values (a higher value indicates greater significance) and the $\mathrm{x}$ axis is $\log 2$ fold change or the difference in abundance between two experimental groups. The dashed red-line shows where $\mathrm{p}=0.01$ with points above the line having $P<0.01$ and points below the line having $P>0.01$. (b) Chromatograms of structures indicate by black arrows in the volcano plot. 
a

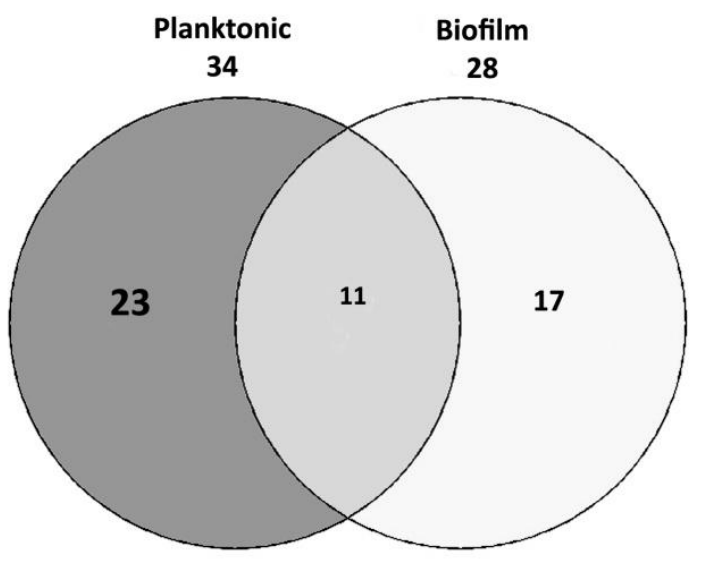

b

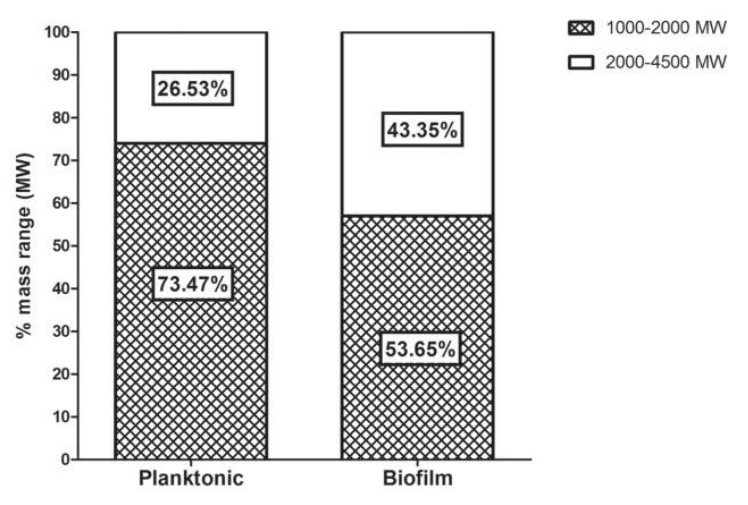

Figure 2. Actinobacillus pleuropneumoniae biofilm and planktonic lipid A structures distribution. (a) Venn diagram showing the differential abundance of lipid A structures between planktonic and biofilm cells with two fold change or higher and $P$ value $>0.01$. (b) Distribution of small (1000-2000 Da) and large (2000-4500 Da) molecular weight (MW) lipid A structures in biofilm and planktonic cells. 
a

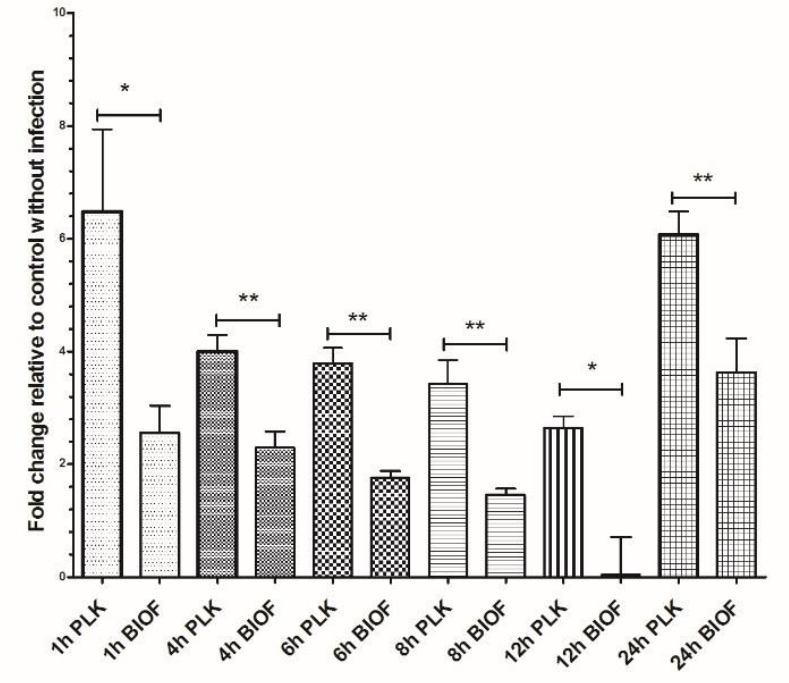

C

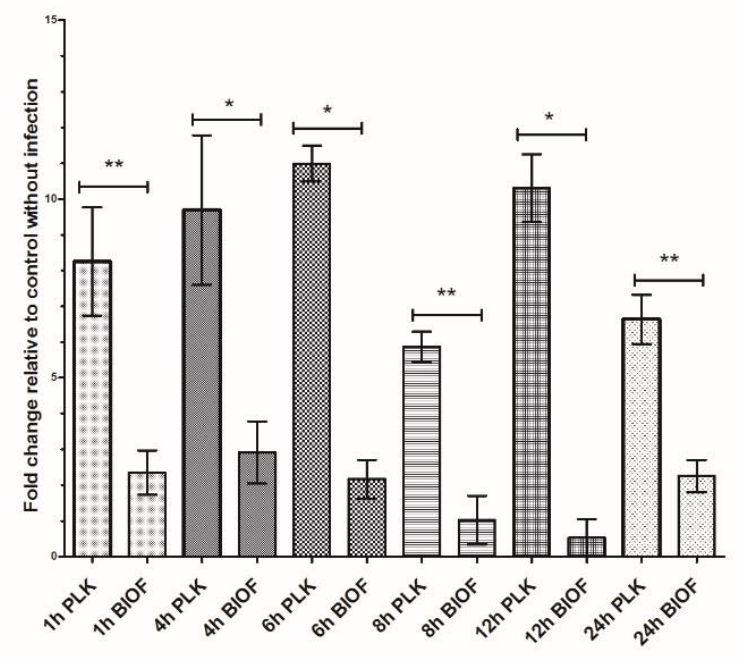

b

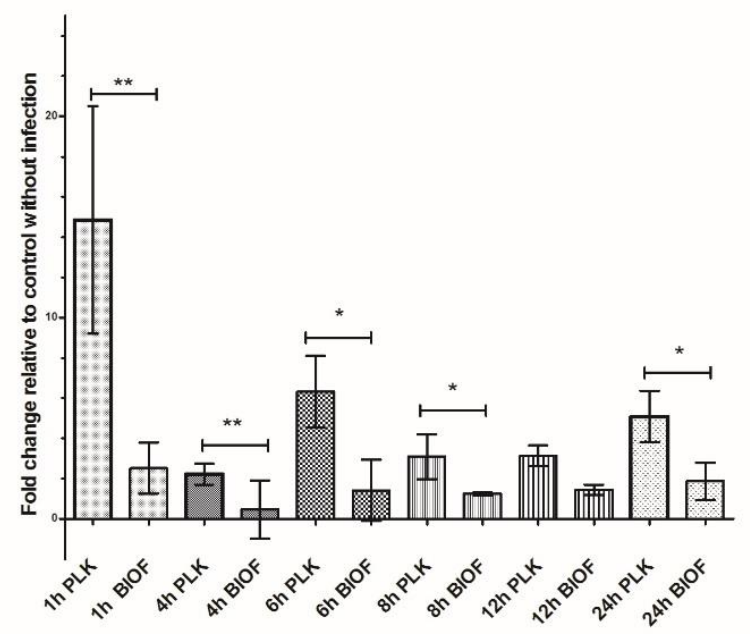

d

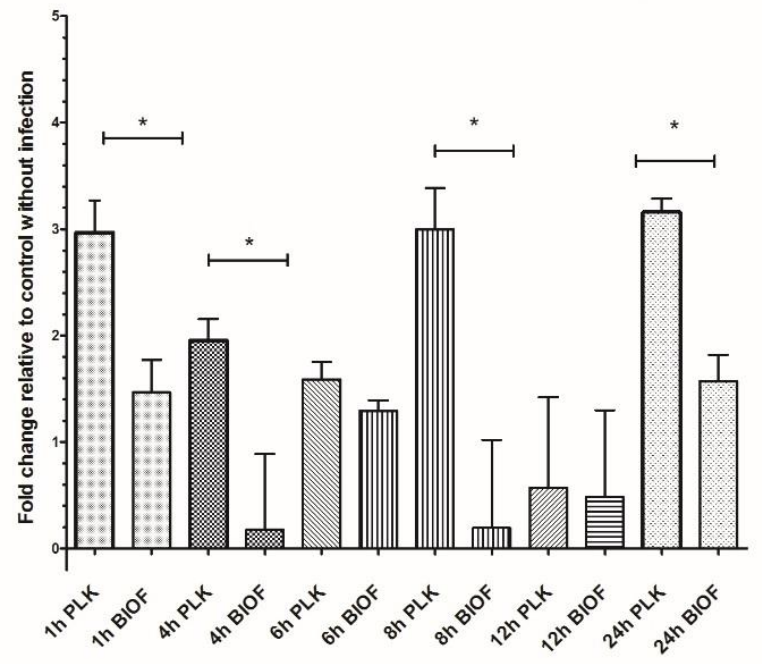

Figure 3. Cytokine expression in porcine pulmonary alveolar macrophages exposed to biofilm (BIOF) or planktonic (PLK) cells of A. pleuropneumoniae (MOI 10:1) for different time periods (1h to $24 \mathrm{~h}$ ). Fold changes in mRNA expression of (a) IL-1, (b) IL-6, (c) IL-8 and (d) MCP-1 relative to the uninfected control are presented. The means \pm SD from 3 independent experiments are shown. Statistical analyses were performed using $2^{-\Delta \Delta \mathrm{Ct}}$ values and all results with $(*)$ or $(* *)$ were statistically significant $(P<0.05$ or $P<0.01$ respectively). 


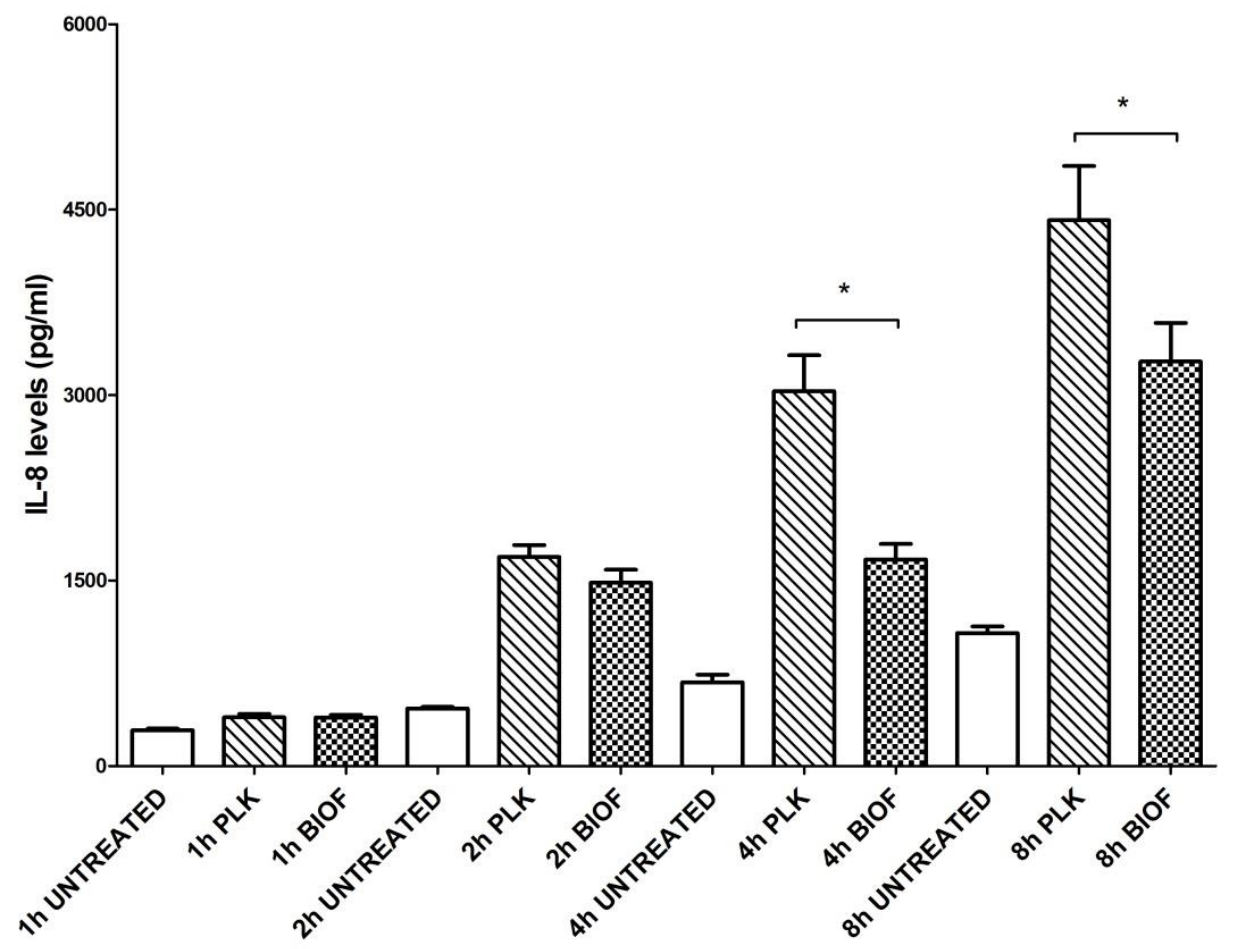

Figure 4. IL-8 production by PAMs in response to stimulation by planktonic (PLK) and biofilm (BIOF) cells (MOI 10:1) for different time periods (1h to $8 \mathrm{~h}$ ). Data are expressed as mean \pm SD (in $\mathrm{pg} / \mathrm{ml}$ ) from at least three independent experiments and results with $(*)$ were statistically significant $(P<0.05)$. 


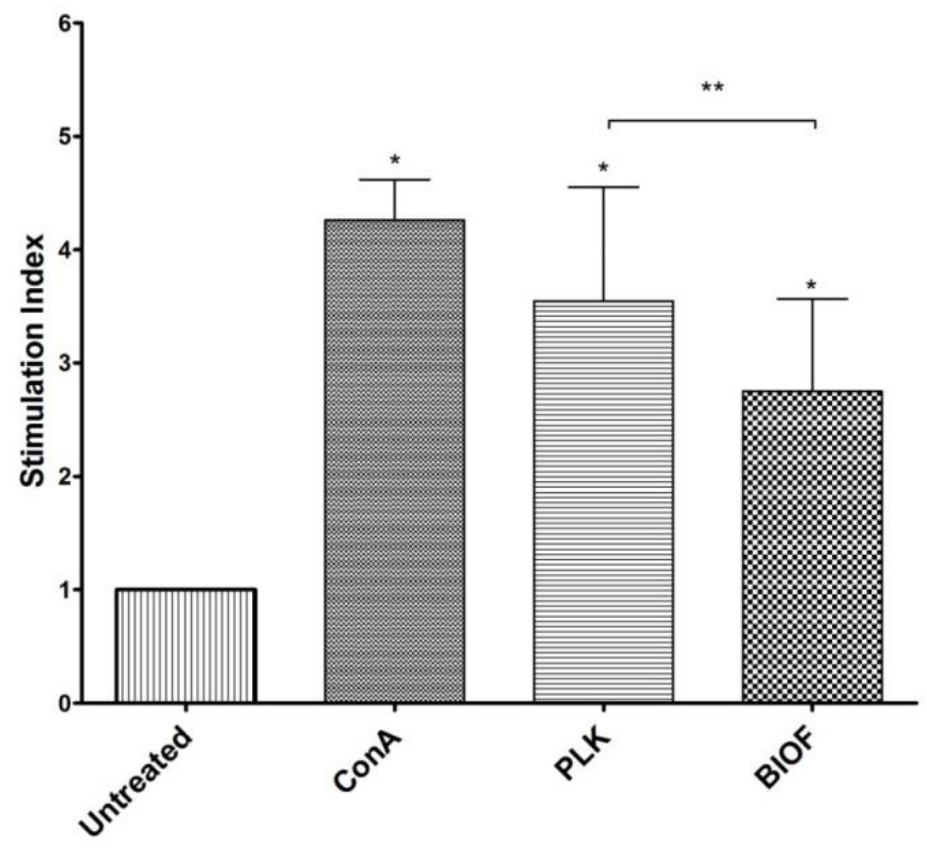

Figure 5. Proliferation of porcine peripheral blood monocytes exposed to biofilm and planktonic cells of A. pleuropneumoniae $\left(2 \times 10^{7}\right.$ cells $\left./ \mathrm{mL}\right)$. Proliferation rate in PBMCs stimulated with concanavalin A (ConA), planktonic (PLK) and biofilm cells (BIOF) of A. pleuropneumoniae was evaluated using a BrdU-ELISA assay. The means \pm SD from 3 independent experiments are shown. $(*)$ or $(* *)$, results were statistically significant $(P<0.05)$. 